\title{
In Vivo Electroporation Mediated Gene Delivery to the Beating Heart
}

\author{
Erick L. Ayuni ${ }^{1}$, Amiq Gazdhar ${ }^{2}$, Marie Noelle Giraud ${ }^{1}$, Alexander Kadner ${ }^{1}$, Mathias Gugger ${ }^{3}$, Marco \\ Cecchini $^{4}$, Thierry Caus ${ }^{1}$, Thierry P. Carrel ${ }^{1}$, Ralph A. Schmid ${ }^{2 *}$, Hendrik T. Tevaearai ${ }^{1}$
}

1 Department of Cardiovascular Surgery, University Hospital of Berne, Berne, Switzerland, 2 Division of General Thoracic Surgery, University Hospital of Berne, Berne, Switzerland, 3 Department of Pathology, University of Berne, Berne, Switzerland, 4 Department of Urology, University Hospital of Berne, Berne, Switzerland

\begin{abstract}
Gene therapy may represent a promising alternative strategy for cardiac muscle regeneration. In vivo electroporation, a physical method of gene transfer, has recently evolved as an efficient method for gene transfer. In the current study, we investigated the efficiency and safety of a protocol involving in vivo electroporation for gene transfer to the beating heart. Adult male rats were anesthetised and the heart exposed through a left thoracotomy. Naked plasmid DNA was injected retrograde into the transiently occluded coronary sinus before the electric pulses were applied. Animals were sacrificed at specific time points and gene expression was detected. Results were compared to the group of animals where no electric pulses were applied. No post-procedure arrhythmia was observed. Left ventricular function was temporarily altered only in the group were high pulses were applied; CK-MB (Creatine kinase) and TNT (Troponin T) were also altered only in this group. Histology showed no signs of toxicity. Gene expression was highest at day one. Our results provide evidence that in vivo electroporation with an optimized protocol is a safe and effective tool for nonviral gene delivery to the beating heart. This method may be promising for clinical settings especially for perioperative gene delivery.
\end{abstract}

Citation: Ayuni EL, Gazdhar A, Giraud MN, Kadner A, Gugger M, et al. (2010) In Vivo Electroporation Mediated Gene Delivery to the Beating Heart. PLoS ONE 5(12): e14467. doi:10.1371/journal.pone.0014467

Editor: Pieter H. Reitsma, Leiden University Medical Center, Netherlands

Received May 24, 2010; Accepted November 10, 2010; Published December 30, 2010

Copyright: (c) 2010 Ayuni et al. This is an open-access article distributed under the terms of the Creative Commons Attribution License, which permits unrestricted use, distribution, and reproduction in any medium, provided the original author and source are credited.

Funding: This work was supported by a grant from the Olga Mayenfisch Foundation, Zurich Switzerland and by two grants from the Swiss National Science Foundation (3200-068304- 2006 to RAS and 310000-118270-2007 to AK).The funders had no role in study design, data collection and analysis, decision to publish, or preparation of the manuscript.

Competing Interests: The authors have declared that no competing interests exist.

*E-mail: ralph.schmid@insel.ch

\section{Introduction}

Coronary artery disease continues to be a major cause of morbidity and mortality worldwide. Currently, the medical treatment and/or myocardial revascularization procedure, either by percutaneous angioplasty or by coronary bypass surgery (CABG) remains the standard therapy [1]. Regeneration of the infracted myocardium, however, is the major challenge and its lack remains the predominant cause of death. New therapeutic modalities which may yield better and consistent results have to be evaluated. Gene therapy, the transfer of nucleic acids to achieve therapeutic benefits is one promising approach. However, progress towards effective human gene therapy in cardiovascular diseases has been hindered by a number of problems including vector toxicity, poor targeting of diseased tissues, and host immune and inflammatory activity [2,3,4]. Hence, safe and reproducible methods have to be established. Electroporation a physical method of gene transfer is one such promising technique.

It is based on the application of strong electric pulses for a very short duration to enhance transfer of macromolecules like DNA and proteins through cell membranes. Even though a number of hypothesis regarding the cell membrane permeabilization have been suggested $[5,6]$, the exact mechanism remains unclear. Nevertheless, electroporation has been shown to be one of the most efficient gene transfer strategies in vivo $[7,8]$ and was tested in a broad range of target tissues and organs [9,10,11,12,13,14]. Ex vivo electroporation to the heart in experimental models has also been reported $[15,16]$ and showed a 100 to 1000 folds increase in transgene expression as compared to direct DNA injection. Consequently, the use of in vivo electroporation mediated transfer may represent a promising method to overcome the common limitations of gene therapy protocols using other types of vectors. Regarding cardiac gene delivery more specifically, direct injection into the cardiac muscle is the most common approach. Gene therapy protocols also involve antegrade coronary injection after aortic cross clamping, however, both approaches do not result in global vector distribution in the heart. Furthermore, the antegrade approach is also limited by heterogeneous and inefficient distribution in the presence of coronary stenosis or occlusion. To overcome this problem we present a retrograde approach by injection via the coronary veins after transient occlusion of the coronary sinus, to study the efficiency and the effect of in vivo electroporation mediated gene transfer to the beating heart.

\section{Materials and Methods}

\section{Plasmid}

The plasmids pCiKlux expresses firefly luciferase from the CMV immediate early promoter/enhancer as described before $[13,17]$. EGFP plasmid was from Add gene (USA). The endotoxin-free plasmids were produced in large scale at Plasmid Factory GmbH \& Co (Bielefeld, Germany). For electroporation, plasmids were suspended in endotoxin-free water. 


\section{Animals}

Inbreed male Wistar rats (200-220 g) (Janvier, France) were used and maintained on rodent feed and water in a air and temperature controlled room. The experiments were performed in compliance with the standards of the European convention of animal care. The study protocols permission numbers 32/03 and 21/06, were approved by the University of Berne Animal Studies Committee.

\section{Gene Transfer technique}

Adult male rats were anesthetized by inhalation of $4 \%$ isoflurane in a glass chamber before being intubated and ventilated via a $14 \mathrm{GA}$ catheter (Insyte, Madrid, Spain) with $\mathrm{FIO} 2=1.0$ and $1.5 \%$ isoflurane to maintain anesthesia, a breathing frequency of $100 / \mathrm{min}$, and a tidal volume of $10 \mathrm{ml} /$ $\mathrm{kg}$ body weight with a rodent ventilator (model 683 Harvard Apparatus, South Natick MA).

A left thoracotomy in the 4th intercostal space was performed and the heart was immobilized, the apex was lifted using an apical 7-0 prolene suture (Ethicon) and a 6-0 tourniquet was placed around the distal coronary vein to allow temporary coronary sinus occlusion (Figure 1a), immediately followed by the retrograde injection of plasmid solution in a volume of $150 \mu \mathrm{l}$ (plasmid concentration $1 \mathrm{mg} / \mathrm{ml}$ ). Animals in all groups below were injected with equal volume of plasmid. Plate electrodes $(2 \mathrm{~cm} \times 1 \mathrm{~cm})$ were placed on each side of the heart followed by the application of 8 pulses of $20 \mathrm{~ms}$ and $200 \mathrm{~V} / \mathrm{cm}, 1 \mathrm{~Hz}$ using the pulse generator (Inovio, San Diego) (Figure 1b). The distance between the two plates of electrode was $1.2 \mathrm{~mm}$. The tourniquet was then immediately released. After the procedure, the heart was observed for 2 minutes before a small chest drain was inserted into the left hemithorax and the thoracotomy was closed with four layers of continuous sutures ( $4 / 0$ prolene). The drain was removed after the animals restored spontaneous breathing, followed by extubation.

\section{Experimental design}

Three sets of experiments were performed.

First, to establish the safety of in vivo electroporation as method of gene transfer to the beating heart. The animals underwent gene transfer as described, with different pulsing parameters of 8,16 and 32 pulses. Animals were evaluated for different parameters of toxicity at different time points.

Second, to establish in vivo electroporation as a method of gene transfer to the beating heart. Two groups were studied $(\mathrm{n}=5)$. All animals underwent gene transfer as described. In the control group, the entire procedure was identical with the exception that the animals were not exposed to the electric pulses. Firefly luciferase under control of CMV immediate early promoter/ enhancer was used and the animals were sacrificed 1 day after gene transfer. One animal was injected with $150 \mu \mathrm{l}$ EGFP expression plasmid and electroporated as described above to localize the gene expression.

Third, to evaluate transgene expression over time. All animals underwent gene transfer as described and subgroups were sacrificed at day $1,3,5$ and 7 after gene transfer $(n=4$ per subgroup).As reporter gene firefly luciferase under control of CMV immediate early promoter/enhancer was used.

\section{Analysis of Toxicity}

Haemodynamic Measurements. Rats were anaesthetized with isoflurane. The right carotid artery was exposed and a 1.4French pressure micro-catheter $\left(\right.$ Millar $^{\circledR}$, Mikro-Tip ${ }^{\circledR}$, Millar Instruments, Houston, TX, USA) was inserted into the left ventricle $(\mathrm{LV})$. LV pressure curves were recorded and analyzed (PowerLab system and its Chart 5.2 software, AD Instruments, Spechbach, Germany). Data were recorded before the electroporation procedure (basal), then continuously over a 60 minutes period. Thereafter, in a subgroup of animals, $(n=4)$ data were measured 6 hours following the electroporation.

Gardiac Enzyme measurements. Two early cardiac specific biomarkers, CK-MB (Creatine kinase) and TnT (Troponin T) were used as indicators of myocardial injury. Blood samples were collected 6 hours following the electroporation, centrifuged and the serum was frozen at $-20^{\circ} \mathrm{C}$. Serum TnT and CK-MB were measured using commercial kits (respectively Roche Diagnostics, Basel Switzerland and Meia, Abbott, Baar, Switzerland) according to manufacturer's instructions.

Histological analysis at each time point. Immediately following euthanasia, the heart was placed in a container with $4 \%$
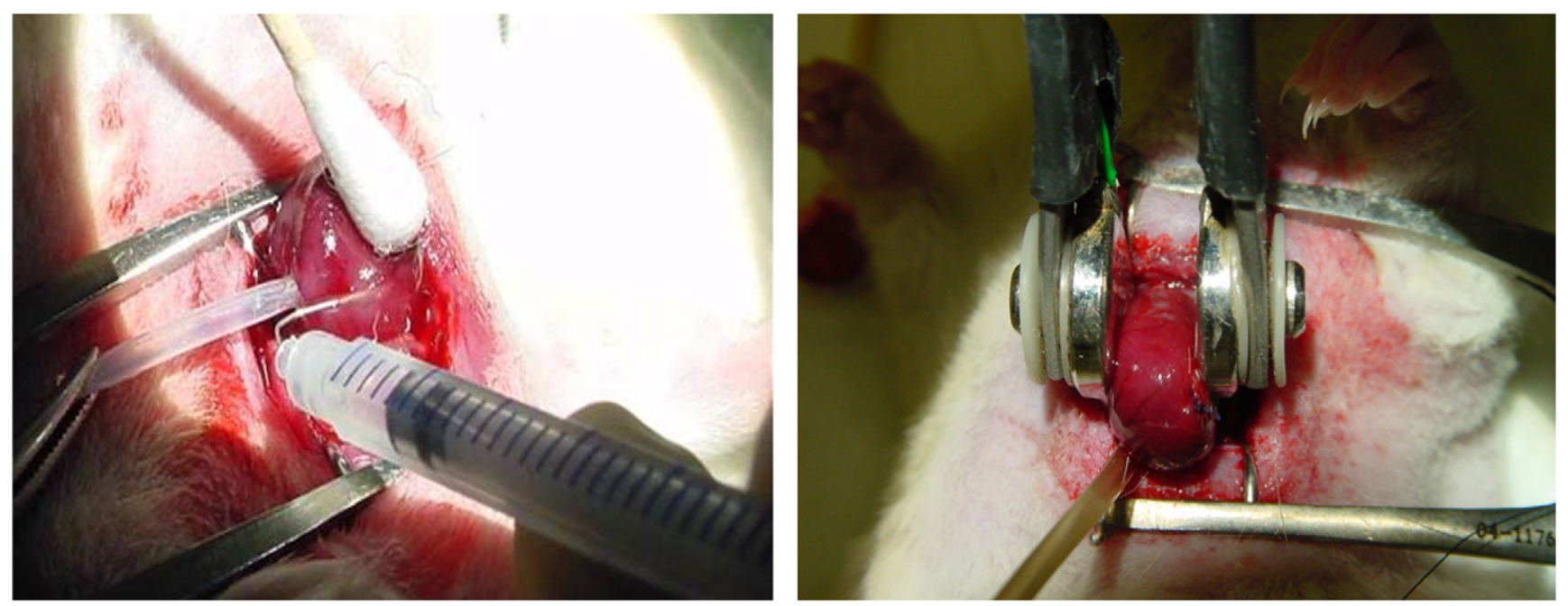

Figure 1. The in vivo electroporation mediated gene transfer to the heart is a two step procedure. (a) First, the coronary sinus is occluded with a 6-0 prolene tourniquet and the plasmid is injected into the coronary vein. (b) The heart is then positioned between the plate electrodes and the electric field is applied.

doi:10.1371/journal.pone.0014467.g001 

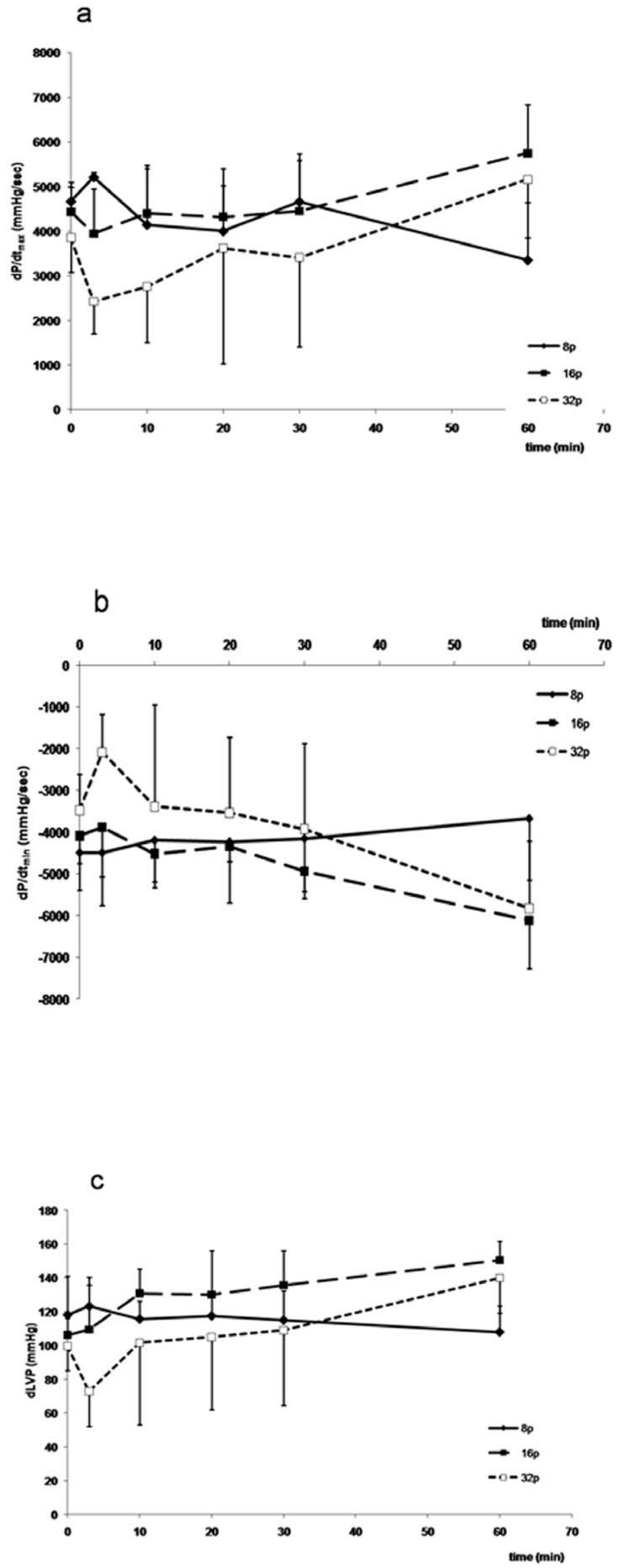
Figure 2. Left ventricular contractile function of heart following electroporation mediated gene transfer using 8, 16 and 32 pulses protocols ( $\mathbf{n}=\mathbf{5}$ each group). Indices of contractility and relaxation determined respectively by the maximum ( $d P / d t$ max) (a) and the minimum (dP/dt min) (b) and developed LVP (dLVP) (c) of the first derivative of ventricular pressure with respect to time. These parameters remained unchanged before (time 0 ) and after electroporation except for the 32 pulses group where the values are transiently altered. A complete normalization of the values occurred within 10-20 minutes. No abnormality was detected in the group in which invivo electroporation was not performed.

doi:10.1371/journal.pone.0014467.g002

formalin for $12 \mathrm{hrs}$. After paraffin embedding the sections where cut and routine haematoxylin and eosin staining was performed. The sections were blinded and reviewed by an experienced pathologist. Gene expression was analyzed at day 1, 3, 5, and 7 following the in vivo electroporation procedure.

Bioluminescent reporter imaging (BLI). The animals were anesthetized with thiopental $(50 \mathrm{mg} / \mathrm{kg}$ Penthotal, Abbot AG, Baar, Switzerland). Monosodium luciferin (750 $\mu \mathrm{l}$ of an $80 \mathrm{mg} / \mathrm{ml}$ solution, Molecular Probes, The Netherlands) was injected i.p, $15 \mathrm{~min}$ before the heart was removed and imaged in an intensified charge-coupled device (CCD) camera (C2400-32, Hamamatsu), fitted with a $50 \mathrm{~mm}$ Nikkor lens (Nikon, Japan) and an image processor (Argus 20, Hamamatsu). An imaging system similar to that described by Contag et al [18] was employed for these studies as previously described [19].

Quantification of the Bioluminescent Signal. The luciferase activity was measured by counting the photon emission in the defined region of interest (ROI) with the aid of the open lab software (Improvision, Coventry, UK) [19].

Measurement of reporter gene expression. Slices of the hearts were frozen in liquid nitrogen immediately after imaging. The tissue was grinded and suspended in $1 \mathrm{ml}$ of lysis buffer (Promega, Madison, WI, USA) and homogenized immediately. Samples were thawed at room temperature and vortexed for $30 \mathrm{sec}$. Subsequently they were refrozen in liquid nitrogen. Three freeze-thaw cycles were performed by alternating between liquid nitrogen and room temperature water bath. Debris was removed by centrifugation. Luciferase activity was then measured and expressed as RLU/mg protein, using the slow glow assay system (Promega, Madison WI) in MiniLumat LB 9506 luminometer (Berthold Technologies, Switzerland).

EGFP reporter gene expression and confocal microscopy. At day one after electroporation mediated gene

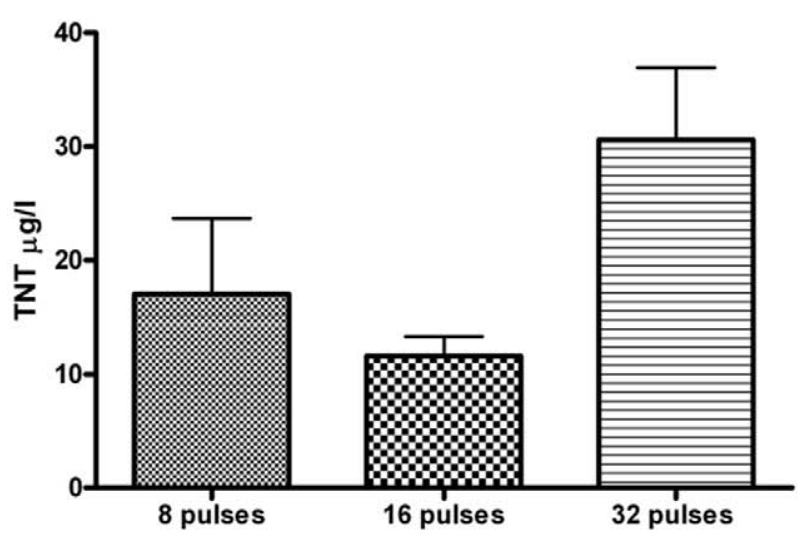

a transfer, the animal was sacrificed and the heart was fixed in O.C.T compound, the cryosections were made at the $10 \mu \mathrm{m}$ thickness and sections were observed under LSM 510 exciter confocal microscope from Carl Zeiss (Germany).

Statistics. Data are presented as mean \pm SEM. The non parametric Mann Whitney U test was performed to compare the groups using GraphPad Prism version 4.00 for Windows, GraphPad Prism version 4.00 for Windows, GraphPad Software, San Diego California USA, www.graphpad.com. $\mathrm{p}<0.05$ was taken as the level of significance.

\section{Results}

Electroporation mediated gene transfer to the beating heart is safe

Electroporation mediated gene transfer to the beating heart is safe Fifty-eight animals were used in the entire study. Overall mortality was $3.4 \%(2 / 58)$, due to bleeding from the coronary vein. Therefore, mortality was never related to the electroporation procedure itself. Especially, mortality remained null among the two groups of animals treated with the higher electroporation doses of 16 and 32 pulses.

Nevertheless, a very transient asystole followed the electroporation in 2/8 animals in the 32 pulses protocol. The asystole resumed spontaneously within 5 seconds. No fibrillation or other rhythm alteration was recorded.

LV contractility function was analyzed over the first hour following the electroporation procedure. Contractility ( $\mathrm{LVdp} / \mathrm{dt}$ $\max$ ) and relaxation ( $\mathrm{LVdP} / \mathrm{dt} \min$ ) as well as the developed LVP (dLVP) were altered after the electroporation procedure only in the group of animals with the maximum pulses regimen of 32 pulses (Figure 2); However, these functional alterations were rapidly reversible and complete normalization occurred within

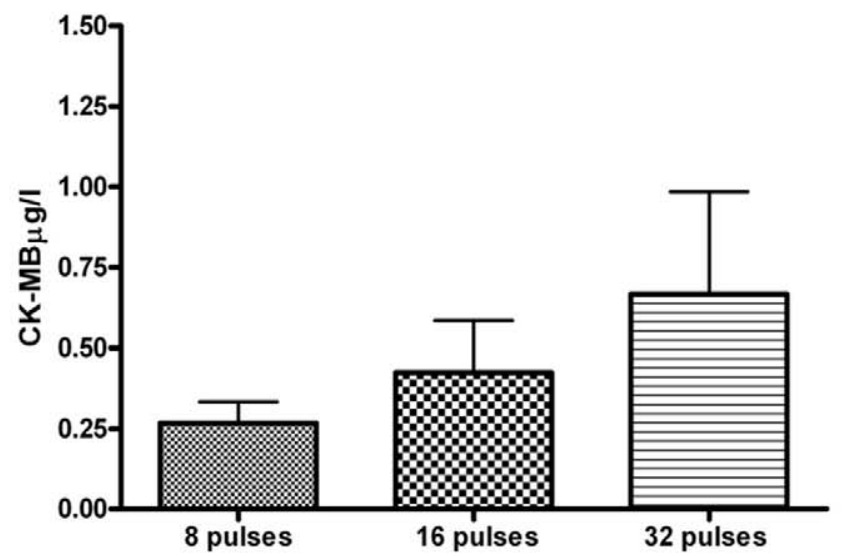

$b$

Figure 3. The two early cardiac enzymes for injury TnT and CK-MB were measured; both enzymes remained within normal range after in vivo electroporation mediated gene transfer with 8 and 16 pulses, but the values were altered with the 32 pulses protocol. (a) TnT levels (b) CK-MB levels. The levels were below detection levels in the group in which invivo electroporation was not performed. doi:10.1371/journal.pone.0014467.g003 
10-20 minutes and persisted thereafter. Conversely, contractile function remained within a normal range in animals' electroporated with either 8 or 16 pulses.

Six hours post-electroporation TNT (Figure 3a) and CK-MB (Figure $3 \mathrm{~b}$ ) values also remained in the normal range in animals treated with 8 and 16 pulses; Values were altered only when a 32 pulses regimen was tested $(30.6 \pm 6.3 \mu \mathrm{g} / \mathrm{l}$ for $\mathrm{TnT}$, and $0.66 \pm 0.318 \mu \mathrm{g} / \mathrm{l}$ for $\mathrm{CK}-\mathrm{MB})$. The levels in the control group without electroporation, were below detection value. Histological analysis performed at each time point, revealed no signs of fibrosis or necrosis however very moderate blood stasis, and very few interstitial lymphocytes, were observed after 8 pulses. For 16 pulses and 32 pulses slightly more blood stasis was observed with some interstitial and perivascular lymphocytes, but no signs of necrosis or fibrosis was seen (Figure 4).

\section{In vivo electroporation mediated gene transfer to the heart increases gene expression}

When $150 \mu \mathrm{l}$ of plasmid DNA pCiklux was injected without electroporation very low transgene expression was detected by BLI imaging (Figure $5 \mathrm{~d}$ ). In contrast, when field strength of $200 \mathrm{~V} / \mathrm{cm}$ and 8 pulses was applied after injection of $150 \mu \mathrm{g}$ of plasmid pCiklux the mean lux activity was easily detected with BLI imaging at day 1 (Figure 5a). The luciferase activity was measured by counting the photon emission in the defined region of interest (ROI), RLU measured were 16479 44338 RLU for electroporated heart vs. $3539 \pm 1555$ RLU for non electroporated heart $(p=0.018)$ (Figure 5b). These findings were further confirmed with conventional luciferase assay in the same samples. Luciferase activity observed on day 1 after electroporation mediated gene transfer was highest; 6594 $\pm 1806 \mathrm{RLU} / \mathrm{mg}$ for electroporated heart vs $1684 \pm 760 \mathrm{RLU} / \mathrm{mg}$ of total protein for non electroporated heart $(\mathrm{p}=0.011)$ (Figure $5 \mathrm{c})$; however, the activity showed a gradual decrease, and at day 7 no transgene expression was seen (Figure 6). The confocal images showed the expression of the EGFP on the cell surface after invivo electroporation mediated gene transfer of the EGFP plasmid (Figure 5 e).

\section{Discussion}

Regenerative medicine is a rapidly evolving field and efficient and reliable methods are needed to expedite the process of regeneration and healing. Current study demonstrates that in vivo electroporation mediated gene transfer to the beating heart is a feasible novel approach; it is also safe, quick, efficient and reproducible. After retrograde injection of plasmid DNA into the transiently occluded coronary sinus and immediate local applica- tion of a series of 8 electric pulses, a high gene expression can be achieved with no obvious adverse effect.

With the shortcomings of viral vectors and the inefficiency of the other non-viral vectors, the physical method of electroporation mediated gene transfer has emerged as a promising tool. Recently the use of this technique in vivo has gained increasing acceptance and has been used successfully in many tissues and organs $[8,11,20]$, under various conditions $[12,14,21,22]$. Even though interest has been shown towards this promising approach, yet its use in the cardiac muscles is limited. Few prior investigations reported the use of electroporation mediated gene transfer to cardiac tissue ex-vivo. Harrisson et al [15] electroporated chick embryonic hearts ex vivo by placing the heart in a bath containing the plasmid DNA, and showed high GFP and luciferase expression after $48 \mathrm{hrs}$. Similarly in the mice heterotopic heart transplant model Wang et al [16] demonstrated that ex vivo electroporation mediated gene transfer of the graft before its implantation, allowed significant gene expression. Very recently this technique was also applied on the pig heart [23]. Therefore, it was with no doubt that electroporation would enhance gene expression in the cardiac muscle. In the current study, however we demonstrate the applicability of this technique with ease and safety on beating hearts of normal rats.

Various routes of administration of the plasmids in the heart have been reported, but none of the protocols could achieve satisfactory global distribution in the heart, especially not in animals with heart failure. Some studies have evaluated the easy direct injection of plasmid into the heart muscles as an efficient method. It is however limited by local needle injury and time consuming electromechanical mapping techniques [24]. The epicardial delivery is another approach where the natural cavity formed by the pericardial pouch was thought to be an advantage. The higher permeability of the pericardium as compared to the epicardium, lets the injected solution diffuse more eccentrically than concentrically [25]. Most recently the percutaneous transluminal approach gathered a lot of attention as specially designed ventricular catheters allow direct injection within the myocardium [26], however, the problem of local needle injury and adequate distribution still remains. In laboratory experiments the coronary delivery approach has been largely used by cross clamping of the ascending aorta and immediate injection of the gene solution to the left ventricular cavity, the solution being delivered to perfuse both coronaries [27]. Unfortunately limitations of this technique involve an acute elevation of the afterload that will further reduce LV function following aortic cross clamping. In the present study we evaluated a retrograde approach where the coronary sinus is clamped transiently before the plasmid solution is injected

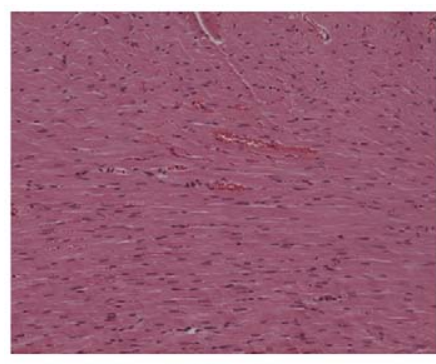

8 pulses

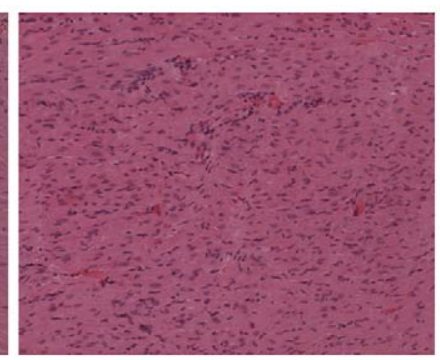

16 pulses

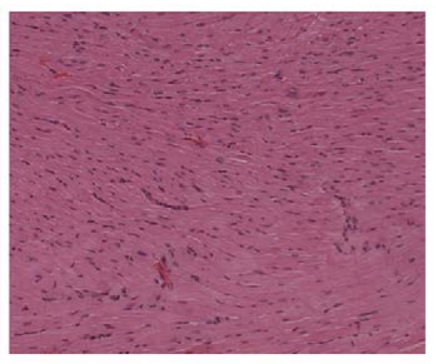

32 pulses

Figure 4. For histological analysis the hearts were evaluated at $\mathbf{2 4}$ hrs post gene transfer. The following criteria were considered: vascular congestion, infiltration and polymorphonuclear infiltrates. No haemorrhage or infiltration was noticed with any pulsing protocol. (a) 8 pulses (b) 16 pulses (c) 32 pulses (Magnification $=\times 200$ ).

doi:10.1371/journal.pone.0014467.g004 
a

\section{Whole heart}
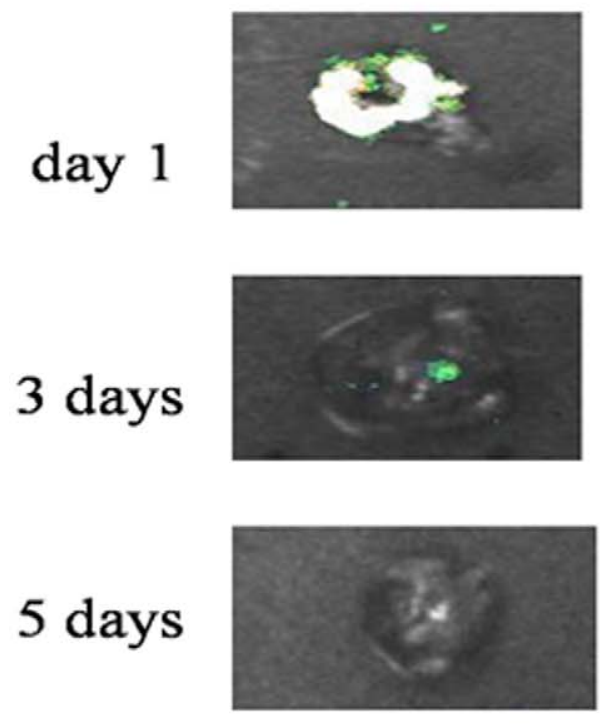

b

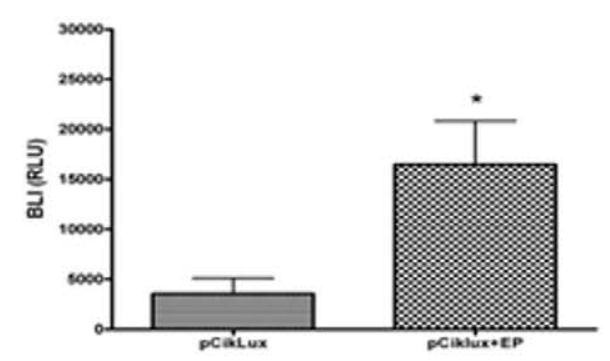

d

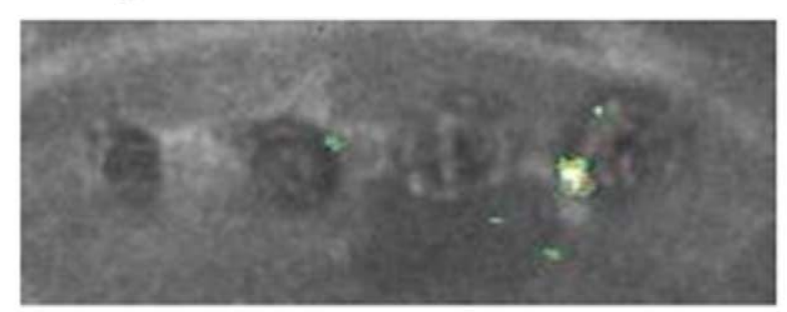

\section{apex middle basis atria}
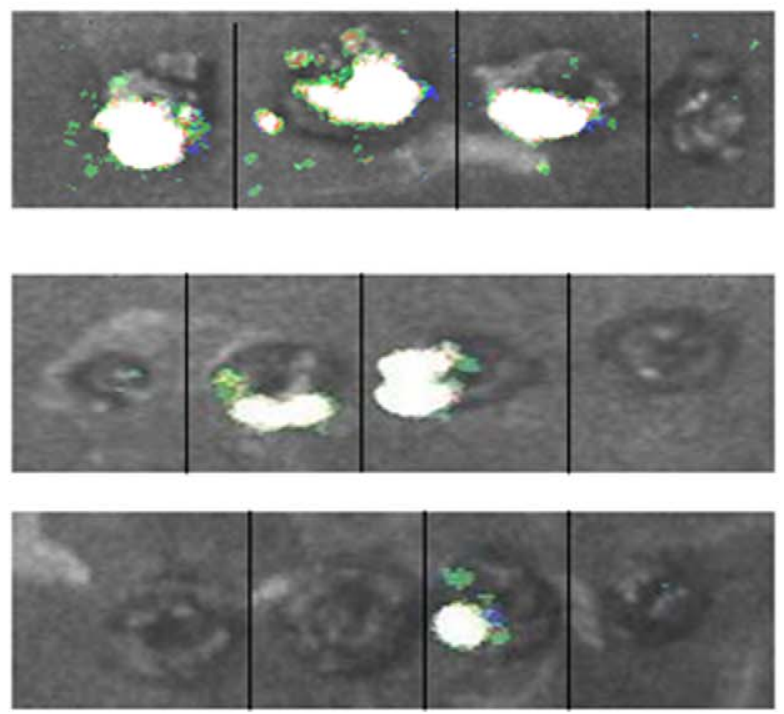

C

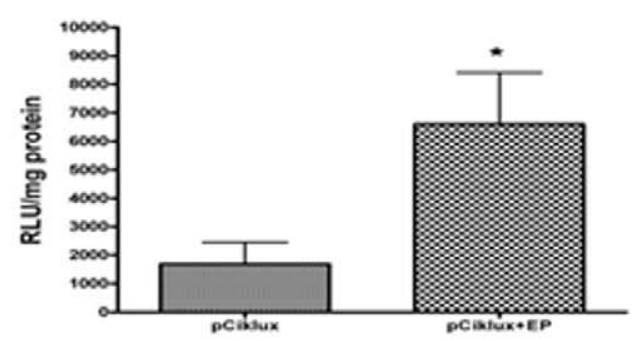

e

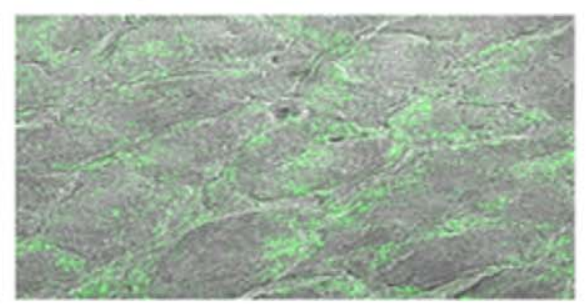

Figure 5. In vivo electroporation mediated gene transfer to the beating heart. (a) BLI images at day 1, 3 and 5 after in vivo electroporation mediated gene transfer to the normal heart. Graphical representation of the quantification of the luciferase activity, with electroporated compared to non-electroporated hearts; (b) BLI measurements, the photon counts per heart field are expressed in relative light units (RLU) $16479 \pm 4338$ RLU for electroporated heart vs. $3539 \pm 1555$ RLU for non electroporated heart $(p=0.018)$ at day 1 . (c) Luciferase assay, RLU per milligram (mg) of protein was measured using a luminometer in the same sample $6594 \pm 1806 \mathrm{RLU} / \mathrm{mg}$ for electroporated heart vs $1684 \pm 760 \mathrm{RLU} / \mathrm{mg}$ of total protein for non electroporated heart $(p=0.011)$ at day 1. (d) Control heart, plasmid was injected but not elecroporated. (e) Expression of EGFP was found on the cell surface.

doi:10.1371/journal.pone.0014467.g005

retrograde into the coronary veins. Using this approach, we were able to achieve global transgene expression in the heart, hence opening new ways of delivering genes to failing hearts.

Since application of direct defibrillation to the heart is almost a routine procedure at the end of cardiac surgery procedures, hence in vivo electroporation mediated gene transfer to the beating heart could easily be applied intraoperatively in routine clinical practice. Numerous studies of myocardial damage caused by electrical currents have been performed [28] and experiments have been conducted to evaluate the toxic effect of electroporation mediated gene transfer to the myocardium [29,30]. It has been shown recently that electroporation induces only very transient phenotypic 


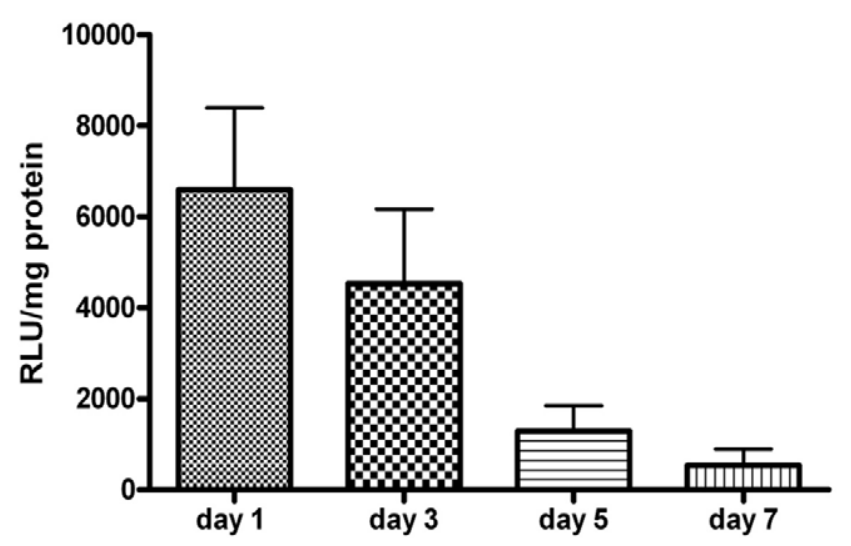

Figure 6. Graphical representation of transgene expression measured over time. After electroporation mediated gene transfer the animals were sacrificed at different time points. doi:10.1371/journal.pone.0014467.g006

and morphological alterations of skeletal muscle fibres [31]. In our current study, we also systematically analyzed the possible side effects of in vivo electroporation on the cardiac muscle including biochemical, functional and histological parameters. In the group treated with 8 and 16 pulses, no alteration in LV function was observed; transient asystole and reduced contractile function as well as slightly elevated levels of TNT and CK-MB were observed only when 32 pulses were administered. Histological assessment revealed no signs of injury, haemorrhage or infiltration.

\section{References}

1. Freedman SB, Isner JM (2002) Therapeutic angiogenesis for coronary artery disease. Ann Intern Med 136: 54-71.

2. Byrnes AP, Rusby JE, Wood MJ, Charlton HM (1995) Adenovirus gene transfer causes inflammation in the brain. Neuroscience 66: 1015-1024.

3. Marshall E (1999) CLINICAL TRIALS: Gene Therapy Death Prompts Review of Adenovirus Vector 10.1126/science.286.5448.2244. Science 286: 2244-2245.

4. Mitani K, Kubo S (2002) Adenovirus as an integrating vector. Curr Gene Ther 2: $135-144$.

5. Neumann ES, Sowers AE, Jordan CA (1989) Electroporation and Electrofusion in Cell Biology. New York: Plenum Press.

6. Gehl J, Sorensen TH, Nielsen K, Raskmark P, Nielsen SL, et al. (1999) In vivo electroporation of skeletal muscle: threshold, efficacy and relation to electric field distribution. Biochim Biophys Acta 1428: 233-240.

7. Mathiesen I (1999) Electropermeabilization of skeletal muscle enhances gene transfer in vivo. Gene Ther 6: 508-514.

8. Mir LM, Bureau MF, Gehl J, Rangara R, Rouy D, et al. (1999) High-efficiency gene transfer into skeletal muscle mediated by electric pulses. Proc Natl Acad Sci U S A 96: 4262-4267.

9. Aihara H, Miyazaki J (1998) Gene transfer into muscle by electroporation in vivo. Nat Biotechnol 16: 867-870.

10. Blair-Parks K, Weston BC, Dean DA (2002) High-level gene transfer to the cornea using electroporation. J Gene Med 4: 92-100.

11. Heller R, Jaroszeski M, Atkin A, Moradpour D, Gilbert R, et al. (1996) In vivo gene electroinjection and expression in rat liver. FEBS Lett 389: 225-228.

12. Nunamaker EA, Zhang HY, Shirasawa Y, Benoit JN, Dean DA (2003) Electroporation-mediated delivery of catalytic oligodeoxynucleotides for manipulation of vascular gene expression. Am J Physiol Heart Circ Physiol 285: H2240-2247.

13. Gazdhar A, Bilici M, Pierog J, Ayuni EL, Gugger M, et al. (2006) In vivo electroporation and ubiquitin promoter - a protocol for sustained gene expression in the lung. J Gene Med 8: 910-918.

14. Tavakoli R, Gazdhar A, Pierog J, Bogdanova A, Gugger M, et al. (2006) Electroporation-mediated interleukin-10 overexpression in skeletal muscle reduces acute rejection in rat cardiac allografts. J Gene Med 8: 242-248.

15. Harrison RL, Byrne BJ, Tung L (1998) Electroporation-mediated gene transfer in cardiac tissue. FEBS Lett 435: 1-5.

16. Wang Y, Bai Y, Price C, Boros P, Qin L, et al. (2001) Combination of electroporation and DNA/dendrimer complexes enhances gene transfer into murine cardiac transplants. Am J Transplant 1: 334-338.

17. Gill DR, Smyth SE, Goddard CA, Pringle IA, Higgins CF, et al. (2001) Increased persistence of lung gene expression using plasmids containing the ubiquitin $\mathrm{C}$ or elongation factor lalpha promoter. Gene Ther 8: 1539-1546.
Although the results are very promising, the technique has a few limitations. Electroporation is a novel approach and needs practice; indeed, the injection has to be performed very diligently since the coronary vein is fragile. There may be concern about the homogenous distribution with retrograde injection, as compared to the antegrade approach. However, the retrograde approach offers the major advantage that it can perfuse ischemic areas whereas the antegrade delivery is limited by coronary stenosis and/or occlusion. For effective gene therapy of chronic disease, persistent transgene expression is needed. In this present study we have used the GMV early promoter enhancer which allows early and transient expression; undergoing transcriptional inactivation $[32,33]$. Further studies are needed with more advanced promoter systems which could provide prolonged transgene expression [13] and also this technique should be tested in infracted heart.

In conclusion, this initial study demonstrates that in vivo electroporation mediated gene transfer to the beating heart is feasible and safe. A high gene expression can be achieved in normal hearts. These observations make in vivo electroporation an attractive alternative to commonly used delivery techniques for gene therapy for heart disease. However, further evaluations including the use of functional genes are required to further evaluate this technique before clinical applications.

\section{Author Contributions}

Conceived and designed the experiments: RAS HTT. Performed the experiments: ELA AG. Analyzed the data: AG MNG AK MG MC. Contributed reagents/materials/analysis tools: MC RAS. Wrote the paper: TC TPC RAS HTT. Corresponding author.

18. Contag CH, Spilman SD, Contag PR, Oshiro M, Eames B, et al. (1997) Visualizing gene expression in living mammals using a bioluminescent reporter. Photochem Photobiol 66: 523-531.

19. Wetterwald A, van der Pluijm G, Que I, Sijmons B, Buijs J, et al. (2002) Optical Imaging of Cancer Metastasis to Bone Marrow: A Mouse Model of Minimal Residual Disease. Am J Pathol 160: 1143-1153.

20. Lin CR, Tai MH, Cheng JT, Chou AK, WangJJ, et al. (2002) Electroporation for direct spinal gene transfer in rats. Neurosci Lett 317: 1-4

21. Lin CR, Yang LC, Lee TH, Lee CT, Huang HT, et al. (2002) Electroporationmediated pain-killer gene therapy for mononeuropathic rats. Gene Ther 9: 1247-1253.

22. Gothelf A, Mir LM, Gehl J (2003) Electrochemotherapy: results of cancer treatment using enhanced delivery of bleomycin by electroporation. Cancer Treat Rev 29: 371-387.

23. Marshall WG, Jr., Boone BA, Burgos JD, Gografe SI, Baldwin MK, et al. Electroporation-mediated delivery of a naked DNA plasmid expressing VEGF to the porcine heart enhances protein expression. Gene Ther 17: 419-423.

24. Vale PR, Losordo DW, Milliken CE, Maysky M, Esakof DD, et al. (2000) Left ventricular electromechanical mapping to assess efficacy of phVEGF(165) gene transfer for therapeutic angiogenesis in chronic myocardial ischemia. Circulation 102: 965-974.

25. Fromes Y, Salmon A, Wang X, Collin H, Rouche A, et al. (1999) Gene delivery to the myocardium by intrapericardial injection. Gene Ther 6: 683-688.

26. Parsa CJ, Reed RG, Walton GB, Pascal LS, Thompson RB, et al. (2005) Catheter-mediated subselective intracoronary gene delivery to the rabbit heart: introduction of a novel method. J Gene Med 7: 595-603.

27. Wright MJ, Wightman LM, Latchman DS, Marber MS (2001) In vivo myocardial gene transfer: optimization and evaluation of intracoronary gene delivery in vivo. Gene Ther 8: 1833-1839.

28. Nikolski VP, Efimov IR (2005) Electroporation of the heart. Europace 7 Suppl 2: 146-154.

29. Bonnafous P, Vernhes M, Teissie J, Gabriel B (1999) The generation of reactiveoxygen species associated with long-lasting pulse-induced electropermeabilisation of mammalian cells is based on a non-destructive alteration of the plasma membrane. Biochim Biophys Acta 1461: 123-134.

30. Rubenstrunk A, Mahfoudi A, Scherman D (2004) Delivery of electric pulses for DNA electrotransfer to mouse muscle does not induce the expression of stress related genes. Cell Biol Toxicol 20: 25-31.

31. Bertrand A, Ngo-Muller V, Hentzen D, Concordet JP, Daegelen D, et al. (2003) Muscle electrotransfer as a tool for studying muscle fiber-specific and nervedependent activity of promoters. Am J Physiol Cell Physiol 285: C1071-1081. 
32. Baskar JF, Smith PP, Nilaver G, Jupp RA, Hoffmann S, et al. (1996) The enhancer domain of the human cytomegalovirus major immediate-early promoter determines cell type-specific expression in transgenic mice. J Virol 70: $3207-3214$.
33. Loser P, Jennings GS, Strauss M, Sandig V (1998) Reactivation of the previously silenced cytomegalovirus major immediate-early promoter in the mouse liver: involvement of NFkappaB. J Virol 72: 180-190. 